\title{
A Review on Opinion Mining through Customer Experiences
}

\author{
Nandini Burade \\ G.H.Raisoni Academy of Engineering \\ Department of Computer Science, \\ and Technology Nagpur
}

\author{
Ankita Kendhe \\ G.H.Raisoni Academy of Engineering \\ Department of Computer Science, \\ and Technology Nagpur
}

\begin{abstract}
:
Internet is fast growing and global system, which is most reliable and efficient. Now a day's social networking is increased due to which interaction of people with each other and share emotions, feedback, views, experiences, opinion and feedback about anything. Also the use of shopping sites i.e. online shopping is increasing tremendously and customers used to give their reviews and feedbacks, which are most important for buying or selling any product. But being as a customer it is difficult to read number of reviews every time while shopping. It leads to create confusion. So the technique which plays an important role in opinion mining and summering reviews of customer is data mining. Some existing techniques for opinion mining show only positive and negative comments from the customer reviews which is not efficient. The main aim of this article is to give an overall idea about the work done in past and about the proposed work.
\end{abstract}

\section{Keywords:}

Opinion mining, reviews, feedback.

\section{INTRODUCTION:}

Online service commonly occurs between parties who have never transacted with each other, in an environment where the service customer often has insufficient knowledge about the service provider, also about the goods and services given. This forces the customer to accept the prior performance risk, i.e. to give payment for goods and services before receiving them, which may leave him in an unsafe position. The customer generally has no chance to try and see products, i.e. to touch the oranges, before he buys. On the other hand, the service provider knows exactly what he gets, so long as the customer is paid in money. The inefficiencies rising from this asymmetry of information can be reduced through trust and reputation. The idea is, even if the customer cannot try the service or product in advance, he can be sure that it will be what he expects as long as he trusts the seller. Therefore the trusted seller has an advantage in case the quality of the product cannot be confirmed in advance.

With the huge growth of social media on the Web, organizations and individuals are mostly using the content in these media for making decisions. For example blogs, reviews, micro-blogs, postings, and comments on social network sites i.e. twitter and Facebook are highly available on web. Nowadays, if anyone wants to buy a customer product, she/he does not depend on family members or

Friends for opinions because many user discussions and reviews are available in public forums about the product on the Web. For an organization, it can no longer be needed to conduct opinion polls, surveys, and to focus on groups in order to collect the public opinions as such information publicly available. Each site generally contains a bulk of opinion text which is difficult to decipher in forum postings and long blogs. It will very difficult to identify the relevant sites and extract or summarize the opinions in those sites for human reader. Thus automated sentiment analysis systems are needed.

Opinions are key influencers of our emotions, views and behavior hence are central to nearly all human activities. We need to know others' opinions whenever we want to take any decision. In the real world, organizations and businesses always need public or customer opinions about their services and products. Similarly individual customer also wants to know the existing user's opinion on a product before purchasing. Previously, whenever anybody needed opinions, she/he asked friends and family. On the other hand a business or an organization collected customer or public opinions by using opinion polls, focus groups and surveys. Opinion mining is the of study which analyzes people's sentiments, opinions, evaluations, emotions and attitudes towards entities such as services, products, organizations, individuals. Opinion is the word that is used to denote sentiment opinion, evaluation, emotion, and attitude. There are also various names for opinion mining but as per name, the task performed by them is somewhat different, e.g., sentiment mining, sentiment analysis, opinion extraction, opinion mining, emotion analysis, subjectivity analysis, review mining, etc. Opinion mining is a type of natural language processing for thinking of the public about a particular product or tracking the sentiment. It is the research area in data mining as well as NLP, Machine Learning, web mining and information retrieval. Opinion mining is useful in many ways. It behaves as market intelligence for organization and business. A huge amount of money is spent to find customer sentiments and opinions. It is the origin which gives information about whether to stop or increase the production of the product. It is also helpful for customer in order to purchase or sale any product. Opinion search supply a search for the opinions, compare two different products and gives opinion on any product.

To extract people's opinion depending on the features of an entity is the important task of opinion mining. For example, the sentence, "The GPS function of Nokia Lumia is good" gives the positive opinion about the "GPS function" of Nokia phone. "GPS function" represents the feature. Another example, "The picture quality of camera is awesome", gives a positive opinion on the camera. "Picture" is the feature. Now how to extract the features is an important problem.

\section{DATA SOURCE:}

Companies and people exploit the unique and rich source of data for variety of purposes. User opinions are the major criterion for the enhancement of the deliverables and to improve the quality services. E commerce sites, review sites, micro blogs and Blogs provide a good understanding of the products and services. 


\subsection{Ecommerce Sites}

E-business is also called as electronic commerce i.e. "ecommerce". For online business activities e-commerce is the wide range of services and products. It is like a business model which enables an individual or a firm to conduct business over an e-network, typically the internet. It is a business transaction where the parties interact with each other electronically. But those parties do not interact by direct physical contact or physical exchanges. E-commerce operates in four segments of market: customer to business, business to business, customer to customer and business to customer. There are many different sites available for e-commerce. Some sites are as follows: Amazon, snap deal, jabong, flipchart etc.

\subsection{Blogs}

A blog is an informational or a discussion site published on the WWW (World Wide Web) and consisting of "posts" typically arranged in reverse chronological order i.e. the current post appears first. Many blogs provide comment on a particular subject; others work as personal online diaries; others function as online advertising of the brand of a company or particular individual. A typical blog combines text, links to other blogs, and images. There are various types of blogs: Media: blog that discuss about any particular media or any media issue only. Political: blog that discuss about any politician or any political issue.

Travel: blog which contains the people reviews about travel agencies or companies and travel experiences with them.

Health: blog which contains people opinion about any hospital or about any disease. Similarly, there are, device blog, educational blog, corporate blog etc.

\subsection{Micro-Blogging}

Micro blogging is broadcast medium in the form of blogging. There is a difference between a micro blog and a traditional blog, in which its content is generally smaller in both aggregate and actual file size. Micro blogs permit users to exchange content with small elements such as video links, short sentences, or individual images. These small messages are called as "micro posts." Micro blogging is the web service which permits the subscribers to post the short messages of the service to each other. Micro posts may be made public on Web site or/and distributed to subscribers private group. Subscribers may request the updates to deliver in real time on their PC as instant message or on a mobile device.

\subsection{Review Sites}

A website onto which reviews are posted about services, products, businesses or people is called a review site. Review sites use the techniques of Web 2.0 to collect reviews from site users or may employ writers to author reviews on the concern topic for the site.

\section{RELATED WORK:}

The following section explains the survey of some papers. Different techniques are used for extracting opinions or sentiments from the given sentences. Following section also explains different techniques which are used to rank and also for classifying these opinions that are extracted from the sentences.

A trust and reputation system [1] is to let parties trust each other on the basis of ratings. Ratings means after completion of transaction leave a comment or a feedback on a product and these ratings will assist the third party in deciding whether or not do transactions in future. Reputation systems are also known as collaborative sanctioning systems. The natural side effect is, it also provides an impetus for good behavior, hence giving the positive results on market quality.

The problems with feature based summarization of reviews of customer about the product that are sold online [2] the set of reviews of a particular product given by the customers is partitioned into three parts. First to identify the features of the product on which customers have expressed their opinions, that is called as features of product. Second for each feature, analyzing review sentences which give negative or positive opinions and ultimately generating a summary using the analyzed information.

The system inputs are an entry page and Product name for all the product reviews. The output is the reviews summery. This method used NLP processor linguistic parser (NLP processor 2000), that identifies simple noun and noun phrases by parsing the search sentence and extracts each word from part of speech. These parts of speech tags are used to find referred frequency features that ultimately give an idea of opinion direction. The disadvantage is, the strength of opinion is not

Shown in this paper. The fundamental technology in current opinion-mining and analysis of sentiments application is classification in two parts [3]:

Part One: covers fundamental background, provides a key concepts discussion involved in common formulations problems of classification in opinion mining and sentiment analysis.

Part Two: covers a depth discussion of various types of classification approaches, ranking problems and regression. It also covers the extraction as it plays an important role in many sentiment oriented applications.

The recently developed natural language processing (NLP) tool and techniques of lexicon based opinion mining are also introduced.

The problem of comparative identification of sentences in text documents [4]-[5]. The problem is similar but somewhat different from sentiment/opinion classification or sentence identification. Identification of comparative sentences is also useful, because the convincing ways of evaluation are direct comparisons, which is more important than individual object opinion. It first divides the comparative sentences into different-different types, and after that the novel integrated pattern is discovered. For identification of comparative sentences from text documents a supervised learning approach is used.

Automatic text summarization [6] for frequent sequences that are found by using multi word, two words or single word patterns. These frequent sequences are known as topic and again the text documents are searched for the group topics and candidate sentences are extracted. The opinions extractions are done using these sentences and ultimately the customer concerns are identified. The use of neural networks in classification of sentiments and proposed an independent model on Artificial neural networks [7] to divide the review corpus into negative, positive and fuzzy tone that is based on an advanced recursive least squares back propagation training algorithm. A neural network based approach, [8] which combines the information retrieval techniques and the advantages of the machine learning techniques. 


\section{CONCLUSION:}

Every organization needs customer opinion to improve their products and to be in competition. All customer compare multiple products at the time of purchasing.

Online prediction is one Web Usage Mining application. Analysis of shopping items uses partial information about the contents of selected shopping items for the prediction of what else the customer is likely to buy. In order to reduce the rule mining cost \& to provide online prediction efficiently, so there is a need to propose architecture for online prediction in Web Usage Mining system and a novel approach for classifying user navigation patterns for predicting users' future requests.

\section{REFERENCES:}

[1] Jøsang, R. Ismail, and C. Boyd, A survey of trust and reputation systems for online service provision, 2007 DSS, vol. 43, no. 2, pp. 618-644, IEEE.

[2] Minqing H., Bing L., Mining Opinion Features in Customer Reviews, Proceedings of the 19th national conference on Artificial intelligence Pages 755-760.

[3] B. Pang and L. Lee, Opinion mining and sentiment analysis Jan. 2008,vol.2, no. 1-2, pp. 1-135,IEEE.

[4] Nitin J., Bing L., Identifying Comparative Sentences in Text Documents 2006, International ACM SIGIR Conference.

[5] Nitin J., Bing L., Mining Comparative Sentences and Relations, national conference on Artificial intelligence Volume 2 Pages 1331-1336.

[6] Jiaming Z., Han T., Ying L., Gather customer concerns from online product reviews- A text summarization approach March, 2009, International Journal Volume 36 Issue 2,. Pages 2107-2115.
[7] ZHU Jian, XU Chen, WANG Han-shi, Sentiment classification using the theory of ANNs, July 2010, The Journal of China Universities of Posts and Telecommunications, IEEE, 58-62.

[8] Long-Sheng Chen, Cheng-Hsiang Liu, Hui-Ju Chiu, A neural network based approach for sentiment classification in the blogosphere, 2011, IEEE.

[9] Shahab Saquib Sohail, Jamshed Siddiqui, Rashid Ali, User Feedback Scoring and Evaluation of a Product Recommendation System, IEEE, 2014

[10] Sheng-Chin Yu, Fong-Ling Fu, Investigating Customer Needs and Evaluation Behavior in online shopping IEEE, 2010 .

[11] Alisa Kongthon, Niran, Chatchawal Sangkeettrakarn, PornpimonPalingoon, ChoochartHaruechaiyasak, Using Opinion Mining Approach to Exploit Web Content in order to improve Customer Relationship Management, IEEE, 2010

[12] Evgeny A.Stepanov, Giuseppe Riccardi, Detecting General Opinions from Customer Surveys, International Conference on Data Mining Workshop, IEEE, 2011.

[13] Raina Agrawal, Pradeep Y., Bridging the Gap between Traditional and Online Shopping Methods for Indian Customers through Digital Interactive Experience, IEEE, 2013.

[14] Mansour Esmaeilpour, Vahideh Naderifar, Predict the customer behavior in the shopping by Distributed Learning Automata, IEEE, 2010.

[15] Ping Sun, Chunhui Wang, A Study of Factors Influencing Customers Online Shopping Behavior based on ANP, IEEE 2010 